\title{
Sensitivity to Instrumental Contingency Degradation Is Mediated by the Entorhinal Cortex and Its Efferents via the Dorsal Hippocampus
}

\author{
Laura H. Corbit, Sean B. Ostlund, and Bernard W. Balleine \\ Department of Psychology University of California, Los Angeles, Los Angeles, California 90095
}

Previous studies have shown that electrolytic lesions of the dorsal hippocampus render the instrumental performance of rats insensitive to selective degradation of the action-outcome contingency (Corbit and Balleine, 2000). In the present experiments, we sought to replicate this finding and to examine the effects of excitotoxic lesions. In the first three experiments, rats with either electrolytic or NMDA lesions of the dorsal hippocampus and sham-lesioned controls were trained to press two levers, each of which delivered a unique food outcome, before their sensitivity to outcome devaluation and degradation of the instrumental contingency was assessed. Although we were able to replicate our original finding that electrolytic lesions of the dorsal hippocampus render rats insensitive to selective degradation of the instrumental contingency, NMDA lesions of the dorsal hippocampus had no effect. Neither lesion had any detectable effect on sensitivity to outcome devaluation.
In experiment 4, we assessed the possibility that the effect of the electrolytic lesion resulted from damage to fibers originating in the retrohippocampal region (including both entorhinal cortex and subiculum) by examining the impact of bilateral NMDAinduced lesions of the retrohippocampus on the same tasks. Importantly, this lesion produced a deficit similar to that observed after electrolytic hippocampal lesions. The final experiment used a disconnection procedure to assess more directly whether damage to efferents from the retrohippocampal region, rather than the dorsal hippocampus itself, can account for the observed deficit. The data from these tests suggest that the deficits observed previously after electrolytic hippocampal lesions were the result of damage to entorhinal efferents.

Key words: entorhinal cortex; dorsal hippocampus; subiculum; instrumental conditioning; outcome devaluation; contingency degradation; reward; context
Current behavioral evidence suggests that, in instrumental conditioning, animals encode the causal relationship between actions and their consequences, as well as the current incentive value of the instrumental outcome (Dickinson and Balleine, 1994; Balleine and Dickinson, 1998). Although the neural structures that mediate these learning processes remain poorly understood, it has been suggested that the hippocampus may be critical for the detection of causal relationships in the environment and that removal of the hippocampus renders rats insensitive to changes in the instrumental contingency, resulting in behavior that is determined primarily by event contiguity (Devenport, 1979, 1980; Devenport and Holloway, 1980).

In a recent study, Corbit and Balleine (2000) examined the effects of hippocampal lesions on encoding the contingent relationship between an instrumental action and its outcome. Rats were trained to press two levers, one delivering sucrose and the other food pellets. After this training, one of the instrumental contingencies was degraded: in addition to the delivery of the sucrose and pellet outcomes contingent on the performance of the instrumental responses, one of these two outcomes was also delivered noncontingently and in such a manner that the probability of the delivery of that outcome was the same whether the action was performed or not. In control rats, this manipulation was found to selectively decrease responding on the lever that

Received June 27; revised Sept. 26; accepted Oct. 1.

This work was supported by National Institute of Mental Health Grant MH 56446 (B.W.B.). We thank Sandra Cetl and Chris Park, who assisted with data collection.

Correspondence should be addressed to Laura Corbit, Department of Psychology, University of California, Los Angeles, Box 951563, Los Angeles, CA 90095. E-mail: corbit@ucla.edu.

Copyright (C) 2002 Society for Neuroscience 0270-6474/02/2210976-09\$15.00/0 delivered the same outcome as that delivered noncontingently. In contrast, rats with electrolytic lesions of the dorsal hippocampus did not show this effect but maintained similar response rates on the two levers, indicating that their performance was relatively insensitive to contingency degradation.

Although these data suggest that the hippocampus is involved in encoding the instrumental contingency, a possible concern is that this study used electrolytic lesions, which have been shown to damage not only cell bodies but also fibers of passage within the lesioned region (Jarrard, 1993). As such, the deficit described above could have been a result of damage to either cells in the dorsal hippocampus or fiber pathways passing through this region.

Given this possibly, experiment 1 sought to replicate our original finding that electrolytic lesions of the hippocampus render rats insensitive to contingency. Experiments 2 and 3 compared the impact of this lesion with the effects of cell body lesions induced by infusions of NMDA into the dorsal hippocampus. The finding that electrolytic but not NMDA lesions of the dorsal hippocampus impair detection of changes in the instrumental contingency suggests that this deficit was a result of damage to fibers of passage.

The retrohippocampal region (i.e., the entorhinal cortex and subiculum) projects heavily through the areas lesioned in experiments 1-3. As a consequence, experiment 4 investigated the effects of NMDA lesions of the retrohippocampus on the sensitivity to contingency degradation. Finally, experiment 5 used a disconnection procedure to determine the relative involvement of the entorhinal cortex and subiculum in this task and to relate the effects of selective lesions of these structures directly to those obtained after electrolytic lesions of the dorsal hippocampus. 


\section{MATERIALS AND METHODS}

\section{Experiment 1}

Subjects and apparatus. The subjects were 20 experimentally naive adult male Long-Evans rats. Rats were housed singly and were handled daily for 1 week before surgery. All procedures used were in accordance with standards established by the Association for Assessment and Accreditation of Laboratory Animal Care for the care of experimental subjects. Training and testing took place in 12 operant chambers (Med Associates, East Fairfield, VT) housed within sound- and light-resistant shells. Each chamber was equipped with a pump fitted with a syringe that delivered $0.1 \mathrm{ml}$ of a $20 \%$ sucrose solution into a recessed magazine in the chamber. Each chamber was also equipped with a pellet dispenser that delivered one $45 \mathrm{mg}$ Noyes pellet (formula A/I) when activated. The chambers contained two retractable levers that could be inserted to the left and right of the magazine and were illuminated by a $3 \mathrm{~W}, 24 \mathrm{~V}$ house light mounted on the top center of the wall opposite the magazine. Microcomputers equipped with the MED-PC program (Med Associates) controlled the equipment, delivered reinforcers, and recorded the lever presses and magazine entries when appropriate.

Surgery and histology. At the time of surgery, rats weighed between 350 and $400 \mathrm{gm}$. The rats were anesthetized with sodium pentobarbital (Nembutal; $50 \mathrm{mg} / \mathrm{kg})$, treated with atropine $(0.1 \mathrm{mg})$, and then placed in a stereotaxic frame with the incisor bar adjusted so that lambda and bregma were level.

Electrolytic lesions. Half of the subjects received electrolytic lesions of the dorsal hippocampus, and the other half received sham surgery that consisted of lowering the electrode into the hippocampus without running any current. Electrodes made from insect pins (size 00) covered in epoxy-polyester varnish except for $1 \mathrm{~mm}$ at the tip were lowered into four sites in the dorsal hippocampus [all coordinates relative to bregma (in $\mathrm{mm}$ ): anteroposterior (AP), -2.8 and -4.2 ; mediolateral (ML), \pm 2.0 and \pm 3.0 ; dorsoventral (DV), -4.0 ). When the electrodes were in place, a 1 $\mathrm{mA}, 20 \mathrm{sec}$ current was passed through the electrode. After surgery, the rats were given 1 week to recover, during which they were handled daily.

Histology. At the end of the experiment, the rats were killed with a lethal barbiturate overdose and perfused transcardially with $0.9 \%$ saline, followed by $10 \%$ Formalin solution. The brains were stored in $10 \%$ Formalin solution for $48 \mathrm{hr}$ and then transferred to a $25 \%$ sucroseFormalin solution before $40 \mu \mathrm{m}$ coronal sections were cut throughout the region of the hippocampus. The slices were stained with thionin and examined for placement and extent of the lesion, with the latter assessed by microscopic examination of sections for areas of marked gliosis.

Procedure. After recovery from surgery, subjects were placed on a food deprivation schedule such that they received $15 \mathrm{gm}$ of their maintenance diet daily to maintain them at $\sim 85 \%$ of their free-feeding weight. During training, the rats were fed after each session. Rats had access ad libitum to tap water while in the home cage. Each session started with the illumination of the house light and insertion of the levers when appropriate and ended with the retraction of the levers and turning off of the house light.

Magazine training. Initially, all subjects received two $30 \mathrm{~min}$ sessions of magazine training in which the two outcomes were delivered on independent random time $60 \mathrm{sec}$ schedules with the levers withdrawn.

Lever training. The rats were first trained with continuous reinforcement (i.e., every action earned an outcome) for $1 \mathrm{~d}$, after which they were placed on a random ratio (RR) 5 schedule (i.e., each action delivered an outcome with a probability of 0.2 ). After $3 \mathrm{~d}$ of training, this was changed to a RR-10 (or a probability of 0.1 ) schedule for $3 \mathrm{~d}$ and then to an RR-20 schedule (or a probability of 0.05 ) for an additional $3 \mathrm{~d}$ of training. The rats received two training sessions each day, one with each actionoutcome pair. The training sessions were $30 \mathrm{~min}$ in duration, and the rats had a break of at least 30 min between sessions.

Contingency degradation training. The instrumental contingency for one of the levers was degraded using a procedure pioneered by Hammond (1980) but as modified in the experiments by Balleine and Dickinson (1998) and Corbit and Balleine (2000). At the end of training, each lever earned a unique outcome (pellets or a $20 \%$ sucrose solution) with a fixed probability, that is, each response had a 1-in-20 chance of delivering the appropriate outcome $\left(p_{(\mathrm{O} / \mathrm{A})}=0.05\right)$. In subsequent sessions, in addition to being earned by one of the actions, one of the outcomes was now also delivered noncontingently with the same probability in each second without a response (i.e., $\left.p_{(\mathrm{O} / \mathrm{no} \mathrm{A})}=0.05\right)$. For one lever, the free reinforcer was the same as that which was earned by a response on that lever. Thus, the experienced probability of the delivery of that particular outcome (e.g., pellets) was the same whether or not the rats performed that action, a procedure that should have acted to degrade that action-outcome contingency. For the other lever, the free reinforcer was different from the earned reinforcer. As such, the rats still had to respond to receive the earned outcome, so this contingency was nondegraded. For half of the rats in each lesion condition, the degraded contingency involved pellet delivery, whereas for the remainder, it involved the delivery of the sucrose solution. The rats had two $20 \mathrm{~min}$ training sessions per day, one on each lever and hence one on each contingency. The rats had a break of $\sim 1 \mathrm{hr}$ between sessions, and the order of the sessions was alternated each day. This training continued for $8 \mathrm{~d}$.

Contingency test. On the day after the final day of contingency training, rats in both groups received a choice extinction test. The test began with the insertion of the levers and the onset of the house light and ended 5 min later with the retraction of the levers and the offset of the house light. No outcomes were presented during this session.

\section{Experiment 2}

The general procedures for this and the following experiments were identical to those described for experiment 1 unless otherwise noted.

Subjects and apparatus. The subjects were 19 experimentally naive male rats that weighed between 450 and $500 \mathrm{gm}$ at the time of surgery.

Surgery and histology: excitotoxic lesions. Half of the subjects received excitotoxic lesions of the dorsal hippocampus, and the other half received sham surgery that consisted of lowering the cannula into the hippocampus without injecting any neurotoxin. Small burr holes were drilled into the skull bilaterally, and cannulas (28 gauge) were lowered into four sites in the dorsal hippocampus [all coordinates relative to bregma (in $\mathrm{mm}$ ): AP, -2.8 and -4.2 ; ML, \pm 2.0 and \pm 3.0 ; DV, -4.0]. A $10 \mu$ l Hamilton syringe was mounted in an infusion pump (Harvard Apparatus, South Natick, MA) and connected to the injection cannulas with polyethylene tubing. Next, $0.4 \mu \mathrm{l}$ of NMDA (20 mg/ml; Sigma, St. Louis, MO) was inf used into the hippocampus at a rate of $0.1 \mu \mathrm{l} / \mathrm{min}$. Five minutes was allowed after the infusion for diffusion before removal of the cannulas. After surgery, the rats were given 1 week to recover, during which they were handled daily.

Procedure. The training and testing procedures were the same as described in experiment 1 above.

\section{Experiment 3}

Subjects and apparatus. The subjects were 19 experimentally naive male rats.

Surgery and histology. The surgical and histological procedures for the electrolytic and excitotoxic lesions were as described in experiments 1 and 2 above.

Procedure. The magazine and lever training procedures used were identical to those described in experiment 1 above. The other procedures were generally similar, with the following exceptions.

Contingency degradation training. Contingency degradation training continued for $4 \mathrm{~d}$, at which point a reliable contingency effect was observed in the NMDA rats. The rats next received a 5 min choice extinction test identical to that described in experiment 1.

Retraining. After the contingency extinction test, the rats received $3 \mathrm{~d}$ of retraining (one session on each lever each day) to recover responding that decreased with the previous test.

Devaluation extinction test. After the final day of retraining, all of the rats were given access ad libitum to one of the two outcomes for $1 \mathrm{hr}$ in the home cage. Half of the rats in each lesion condition received pellets (50 gm placed in a bowl in the home cage), and the remaining rats received sucrose $(50 \mathrm{ml}$ in a drinking bottle fixed to the front of the home cage). Immediately after the prefeeding, the rats were placed in the operant chambers. A 5 min choice extinction test was then conducted in which both levers were extended and the number of presses on each lever was counted. No outcomes were delivered during the test.

\section{Experiment 4}

Subjects and apparatus. The subjects were 24 experimentally naive adult female rats that weighed between 250 and $350 \mathrm{gm}$ at the time of surgery. Surgery. The surgical and histological procedures generally followed those described in experiment 2 above, with the following exceptions.

Excitotoxic lesions. Half of the subjects received excitotoxic lesions of the retrohippocampus, and the other half received sham surgery that consisted of lowering the cannula into the retrohippocampus without injecting any neurotoxin. The coordinates used were adapted from 
A
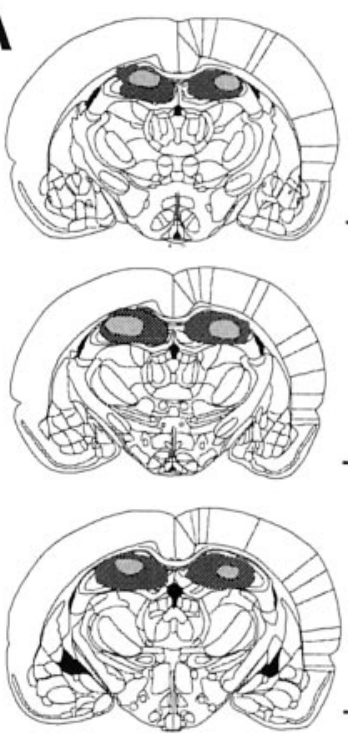

$-3.14$

$-2.56$
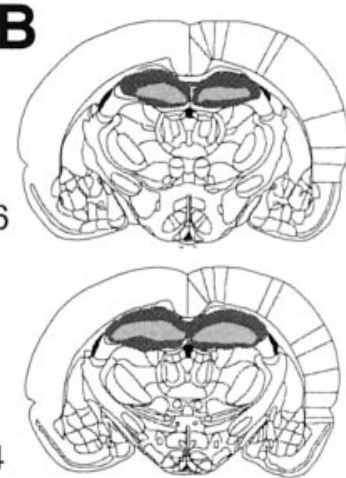

$-3.60$
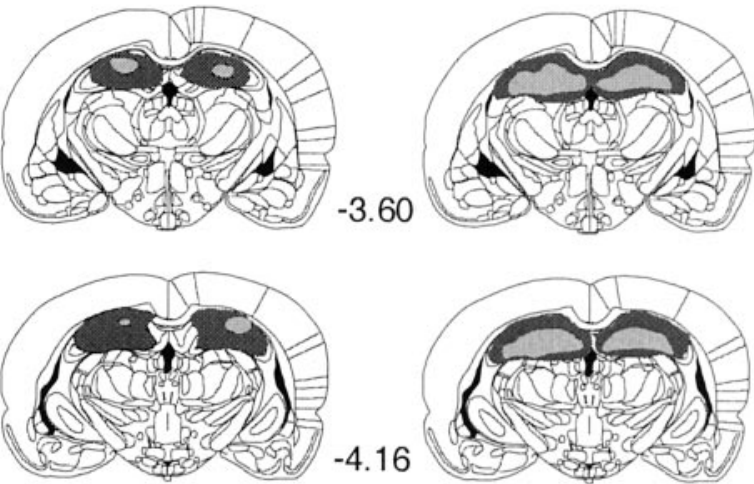

$-4.16$
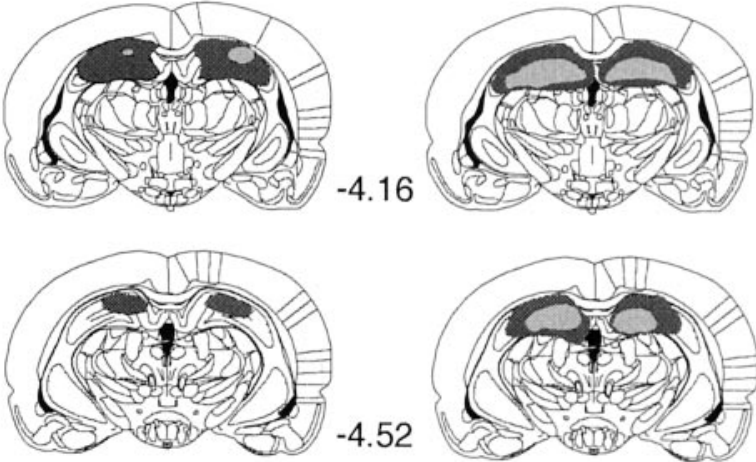

$-4.52$

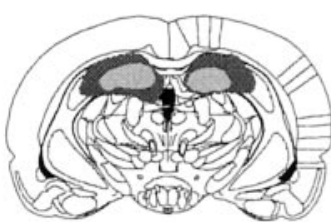

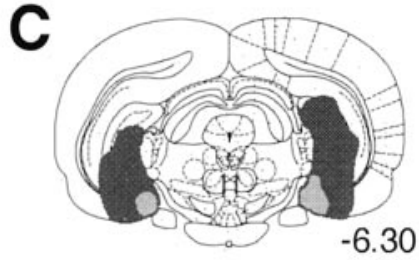

$-6.30$

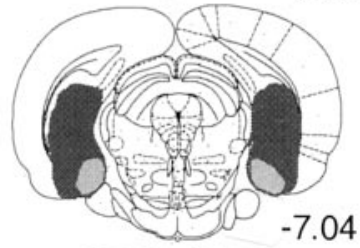

$-7.04$
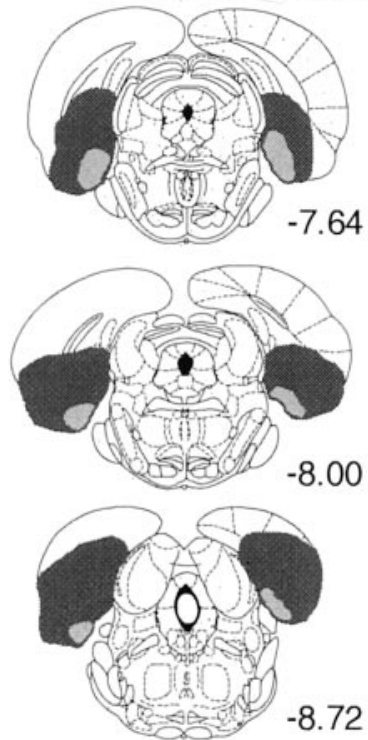

Watson (1998).

Maren et al. (1997), who found that these lesions produced a deficit in context conditioning. Cannulas (28 gauge) were lowered into six sites in the retrohippocampal region [all coordinates relative to bregma (in $\mathrm{mm}$ ): AP, $-6.8,7.5$, and 8.2; ML, $\pm 5.0, \pm 4.5$, and \pm 4.5 ; DV, $-7.0,-6.5$, and $-5.0]$. Next, $0.4 \mu \mathrm{l}$ of NMDA ( $20 \mathrm{mg} / \mathrm{ml}$; Sigma) was infused into each site at a rate of $0.1 \mu \mathrm{l} / \mathrm{min}$ following the procedures outlined in experiment 2 above. One subject in the sham group failed to recover from surgery $(n=11)$.

Procedure. The magazine and lever training procedures were identical to those described in experiment 1 above. The other procedures were generally similar with the following exceptions.

Contingency degradation training. Contingency degradation training continued for $12 \mathrm{~d}$. The rats next received a 5 min extinction test.

Devaluation extinction test and retraining. The devaluation extinction test was identical to that described in experiment 3 .

\section{Experiment 5}

Subjects and apparatus. The subjects were 30 experimentally naive female rats.

Surgery: disconnection lesions. All subjects received unilateral electrolytic lesions of the dorsal hippocampus but were then divided into three groups that received contralateral neurotoxic lesions of either the entorhinal cortex or subiculum or sham surgery that consisted of lowering the cannula into one of these locations without injecting any neurotoxin. The electrolytic lesions were produced as in experiment 1 , except that they were unilateral [left or right side was counterbalanced; all coordinates relative to bregma (in $\mathrm{mm}$ ): $\mathrm{AP},-2.8$ and -4.2 ; $\mathrm{ML}, \pm 2.0$ and \pm 3.0 ; DV, $-4.0]$. Cannulas (28 gauge) were then lowered into two sites in the contralateral entorhinal cortex (in $\mathrm{mm}$ : $\mathrm{AP},-7.5$ and -8.2 ; ML, \pm 4.5 ; $\mathrm{DV},-6.5$ and 5.0) or subiculum (in $\mathrm{mm}$ : AP, -6.3 and -6.8 ; ML, \pm 5.0 ; DV, -6.0 and -4.0$)$. Next, $0.4 \mu \mathrm{l}$ of NMDA $(20 \mathrm{mg} / \mathrm{ml}$; Sigma) was infused into each site at a rate of $0.1 \mu \mathrm{l} / \mathrm{min}$ except in the sham-lesioned group. Five minutes was allowed after the infusion for diffusion before removal of the cannulas. After surgery, the rats were given 1 week to recover, during which they were handled daily.

Procedure. The magazine and lever training procedures were identical to those described in experiment 1 above. The other procedures were generally similar, with the following exceptions.

Contingency degradation training. Contingency degradation training continued for $3 \mathrm{~d}$ and was followed by a 5 min extinction test.

\section{RESULTS}

\section{Histology}

No recovery problem or weight loss was observed after surgery in any of the lesion groups. In all electrolytically lesioned rats (experiments 1 and 3), damage to the dorsal hippocampus was bilateral and complete, except at the rostral and caudal extremes and with some sparing of tissue on the lateral edges of the lesions. Figure 1, left, summarizes the maximum and minimum damage resulting from the lesions for the rats included in the behavioral analysis. No systematic damage to the overlying cortex was observed in either group. All rats receiving excitotoxic hippocampal lesions (experiments 2 and 3 ) had complete bilateral lesions of the dorsal hippocampus, except at the rostral and caudal extremes. Figure 1, middle, summarizes the maximum and minimum damage from the lesions. The lesions were generally consistent between rats. Inspection of the stained tissue did not reveal damage to areas outside the hippocampus in the region of the lesion. In general, these neurotoxic lesions were similar to those obtained with an electrolytic procedure, although the latter produced damage to axonal tracts passing through the dorsal hippocampus, and, 


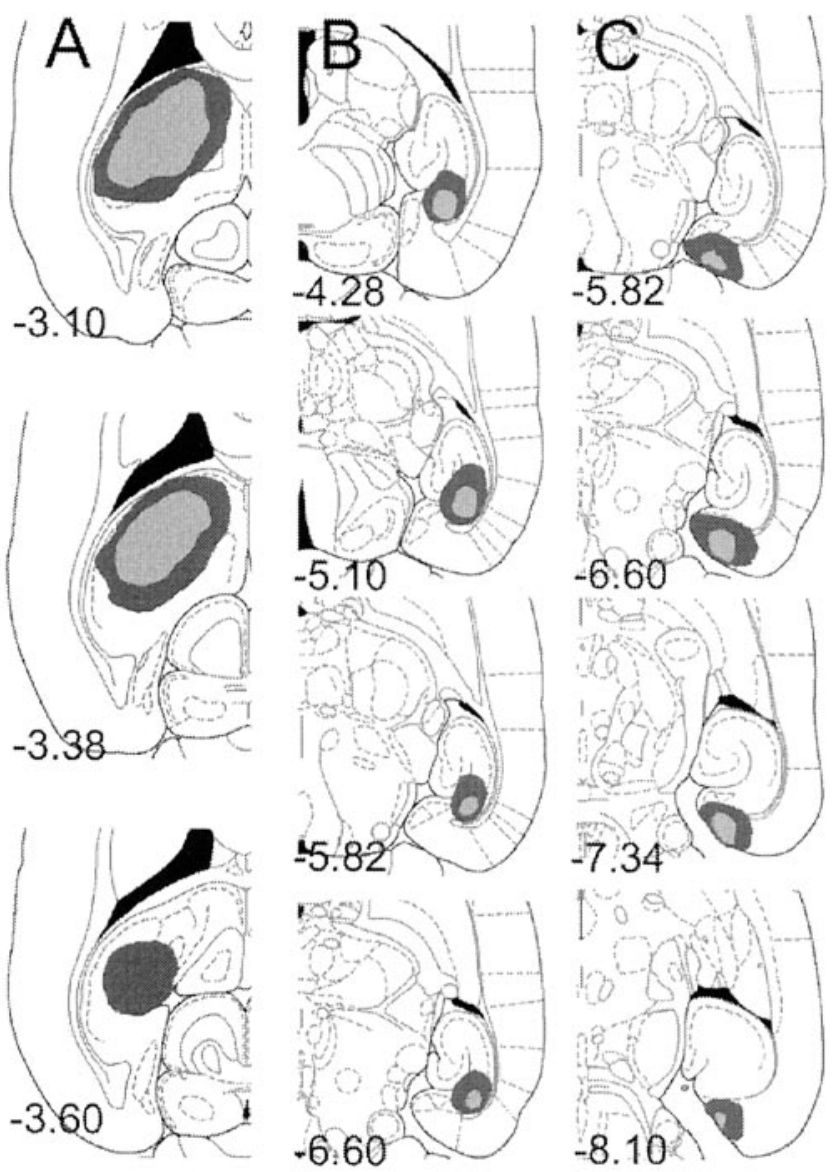

Figure 2. Line drawings of horizontal sections from the brains of subjects with the maximum (dark gray) and minimum (light gray) damage resulting from unilateral electrolytic lesions of the dorsal hippocampus (left) and contralateral (middle) NMDA lesions of subiculum or NMDA lesions of entorhinal cortex (right). Each section is marked with its position in the DV plane (millimeters relative to bregma). Drawings are from Paxinos and Watson (1998).

if anything, electrolytic lesions spared more tissue at the lateral and rostral extremes of the region, whereas NMDA infusions produced a more complete lesion.

All rats receiving excitotoxic lesions of the retrohippocampus (experiment 4) had complete bilateral damage of the medial entorhinal cortex and limited yet reliable damage to the lateral entorhinal cortex, subiculum proper, subicular cortices (presubiculum and parasubiculum), and the caudal aspect of the dentate gyrus. Figure 1, right, summarizes the maximum and minimum damage from the lesions. The lesions were generally consistent between rats.

In experiment 5, unilateral lesions of the dorsal hippocampus were consistent across lesion groups and were similar in extent of damage to electrolytic lesions in experiments 1 and 3 (Fig. 2, left). Contralateral NMDA lesions of the subiculum produced substantial damage to the ventral subiculum. These lesions were often narrow, preserving the medial and lateral portions of the ventral subiculum in several rats. In addition, the coordinates were chosen to minimize the risk of overlap; thus, the most posterior aspect of the ventral structure remained intact in several rats. Animals with large lesions also had limited damage of the presubiculum (Fig. 2, middle), extending rostrally in some rats to the ventral hippocampal fields (dentate gyrus and CA1). Contralat- eral NMDA lesions of the entorhinal cortex (Fig. 2, right) were generally limited to the medial entorhinal cortex but often included some damage to the presubiculum and parasubiculum. Additional investigation would be needed to determine the individual contribution of the presubiculum and parasubiculum in contingency learning. As shown in Figure 2, there was no overlap in the minimal or maximal damage of the subicular and entorhinal lesions.

\section{Experiment 1}

\section{Training}

No effect of the lesion on the acquisition of lever pressing was observed. The two groups acquired the lever press response in the same number of days. Furthermore, no difference in the rate of lever pressing between the lesion [mean responses per minute $(M)=33.43 ; \mathrm{SE}=5.33$ ] and sham $(\mathrm{M}=43 ; \mathrm{SE}=4.40)$ groups was evident at the end of initial training $\left(F_{(1,18)}=1.961 ; p>\right.$ $0.05)$.

\section{Contingency degradation training}

Figure 3, left, shows the effects of contingency degradation across $2 \mathrm{~d}$ blocks of training. Inspection of the figure suggests that, across days, at least in the sham group, the rats showed sensitivity to the degradation of the instrumental contingency, performing more responses on the lever for which the contingency had not been degraded. The statistical analysis of the contingency training data revealed a main effect of contingency $\left(F_{(1,18)}=4.482 ; p<0.05\right)$, a main effect of training day $\left(F_{(3,54)}=5.573 ; p<0.01\right)$, but no main effect of group $(F<1)$. Examination of Figure 3 suggests that the hippocampal rats were not sensitive to the degradation of the instrumental contingency; however, the group $\times$ contingency interaction failed to reach significance $\left(F_{(1,18)}=2.00 ; p>0.05\right)$. None of the other interactions were significant.

\section{Extinction test}

As shown in Figure 3, right, it is clear that the two groups performed very differently on this test. Whereas the sham group showed a clear decrease in responding on the lever for which the contingency had been degraded relative to the other lever, this was not true of the hippocampal rats, which appeared to perform both actions at comparable rates. The analysis of the extinction test data confirmed this description. This analysis revealed no main effect of group $\left(F_{(1,18)}<1\right)$; there was, however, a main effect of contingency $\left(F_{(1,18)}=9.224 ; p<0.01\right)$. Importantly, there was a significant contingency $\times$ group interaction $\left(F_{(1,18)}=\right.$ 7.356; $p<0.01$ ). Simple effects analysis revealed that, for the sham group, there was a significant effect of contingency treatment $\left(F_{(9)}=7.026 ; p<0.01\right)$. As seen in Figure 3, right, these rats performed fewer presses on the lever for which the contingency had been degraded. However, for the lesion group, there was no difference in response rate on the two levers after the contingency treatment $\left(F_{(8)}=1.586 ; p>0.05\right)$.

These results provide additional evidence that electrolytic lesions of the dorsal hippocampus eliminate rats' sensitivity to the selective degradation of the instrumental contingency.

\section{Experiment 2}

\section{Training}

Analysis of the training data revealed that, although the two groups acquired the lever press response in the same number of days, the lesioned rats responded at a higher rate $(M=39.93$; $\mathrm{SE}=4.53)$ at the end of training than did sham rats $(\mathrm{M}=26.67$; 
Figure 3. Mean lever presses per minute across $2 \mathrm{~d}$ blocks of contingency degradation training are depicted at left. For each group, one of the actionoutcome contingencies had been degraded, whereas the other remained nondegraded. Right, Lever responses for electrolytically and shamlesioned rats in a two-lever choice extinction test after the selective degradation of one of the instrumental contingencies. Error bars represent the SE of the difference of the mean $( \pm 1$ SED) for the within-subjects variable in each group.
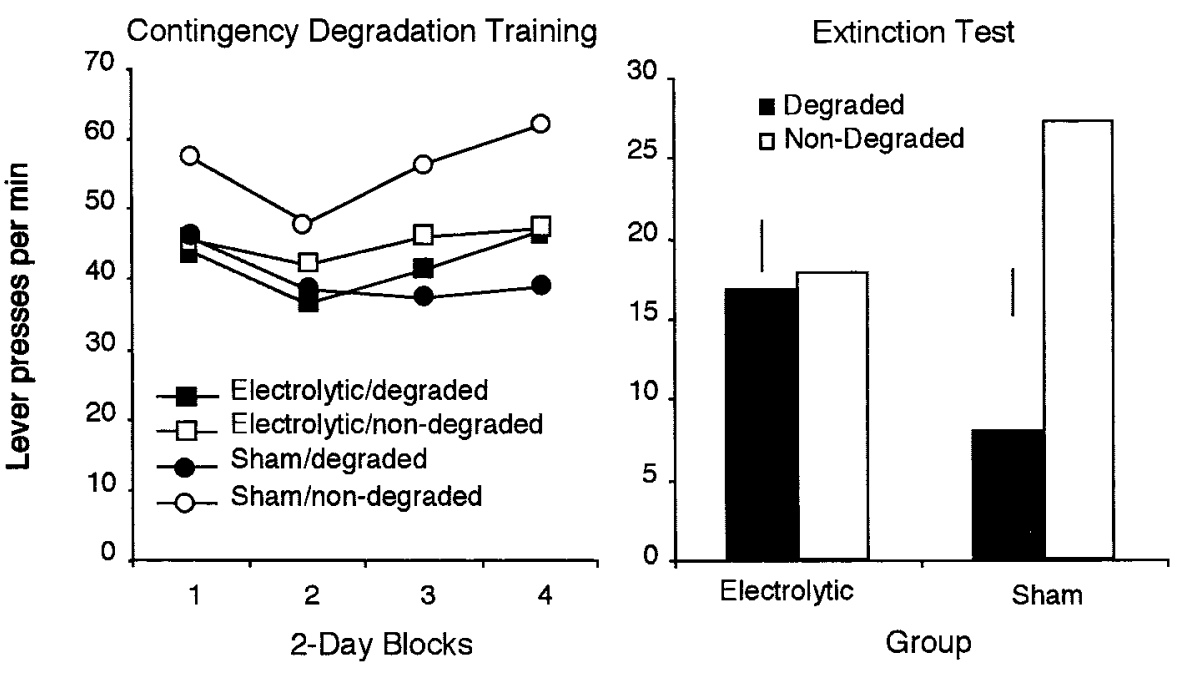

Figure 4. Mean lever presses per minute across $2 \mathrm{~d}$ blocks of contingency degradation training are depicted at left. For each group, one action-outcome contingency had been degraded, whereas the other remained nondegraded. Right, Lever responses for NMDA- and sham-lesioned rats in a two-lever choice extinction test. Error bars represent \pm 1 SED for the within-subjects variable in each group.

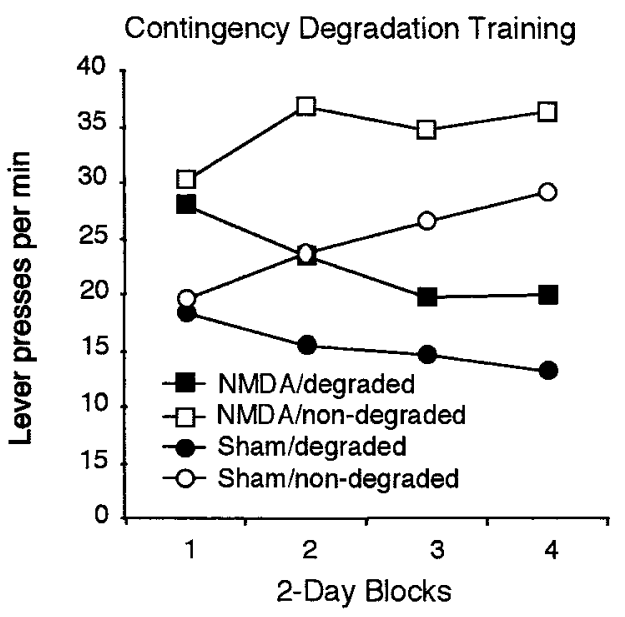

$\mathrm{SE}=3.33)$, and this result was statistically significant $\left(F_{(1,17)}=\right.$ 5.853; $p<0.05)$.

\section{Contingency degradation training}

Inspection of Figure 4, left, suggests that, although response rates were numerically higher in the lesioned rats, the two groups were both sensitive to the contingency manipulation. Analysis of the contingency training data supports this description, revealing no effect of group $\left(F_{(1,17)}=2.286 ; p>0.05\right)$ and no effect of day $(F<$ $1)$. There was, however, a significant effect of contingency $\left(F_{(1,17)}\right.$ $=10.430 ; p<0.01)$ and a day $\times$ contingency interaction $\left(F_{(7,119)}\right.$ $=4.940 ; p<0.01)$, suggesting that the contingency effect grew larger across days. No other interactions reached significance.

\section{Extinction test}

As shown in Figure 4, right, both groups performed fewer responses on the lever for which the contingency had been degraded. Analysis of the extinction test data supports this claim, revealing no effect of group $(F<1)$, a significant effect of contingency $\left(F_{(1,17)}=11.58 ; p<0.01\right)$, and no group $\times$ contingency interaction $(F<1)$. These data suggest that NMDA lesions of the dorsal hippocampus produce little if any impairment in rats' sensitivity to the selective degradation of one action-outcome contingency. This result contrasts markedly with previous findings (Corbit and Balleine, 2000) (experiment 1 above), which have shown that electrolytic lesions of the dorsal hippocampus render rats relatively insensitive to contingency degradation. Given this discrepancy, a more direct comparison of the effects of these two lesion types in a single experiment was conducted in experiment 3 .

\section{Experiment 3}

\section{Training}

There was no difference between groups in acquisition of the lever press response. At the end of training, the electrolytically $(\mathrm{M}=25.30 ; \mathrm{SE}=5.80)$ and NMDA $(\mathrm{M}=27.27 ; \mathrm{SE}=4)$ lesioned rats were responding at similar rates $(F<1)$.

\section{Contingency degradation training}

Figure 5, left, displays the responses for the two groups across days of contingency degradation training. Inspection of the figure suggests that the electrolytically lesioned rats are insensitive to the contingency manipulation. In contrast, rats with NMDA lesions of the hippocampus appear to show normal sensitivity to the contingency degradation manipulation. Statistical analysis of the training data reveals no effect of group $(F<1)$ but a significant effect of day $\left(F_{(3,51)}=5.23 ; p<0.01\right)$ and contingency $\left(F_{(1,17)}=\right.$ $8.72 ; p<0.01)$ and a day $\times$ group interaction $\left(F_{(3,51)}=11.51 ; p<\right.$ $0.01)$; however, despite the numerical trends, the group $\times$ contingency interaction failed to reach significance $\left(F_{(1,17)}=2.19\right.$; $p>0.05)$. No other interactions were significant. 


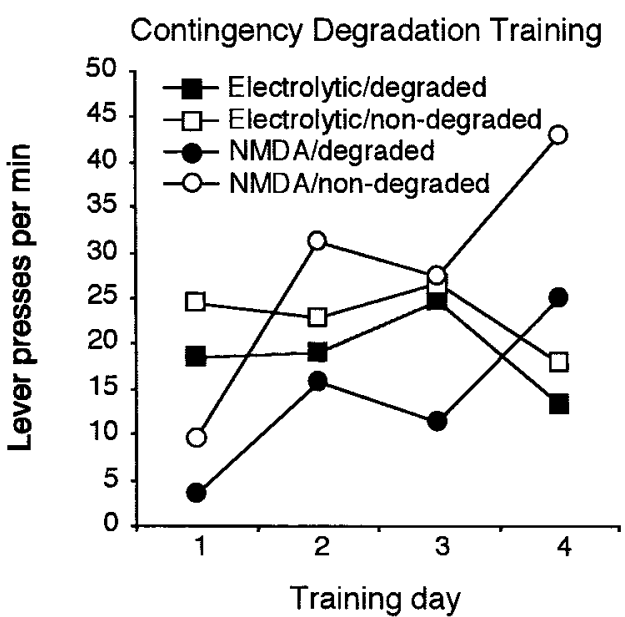

\section{Extinction test}

The data from the extinction test are displayed in Figure 5, right. As suggested by the figure, the rats with NMDA lesions performed fewer responses on the lever for which the contingency had been degraded previously relative to the other lever, whereas the rats with electrolytic lesions responded similarly on the two levers. This description is supported by the statistical analysis, which revealed no effect of group $(F<1)$, a marginal effect of contingency $\left(F_{(1,17)}=3.91 ; p=0.06\right)$, but importantly a group $\times$ contingency interaction $\left(F_{(1,17)}=5.93 ; p<0.05\right)$. Subsequent analysis revealed a contingency effect in the NMDA group but no effect in the electrolytic group. These results confirm that electrolytic and NMDA lesions of the dorsal hippocampus produce different effects with regard to sensitivity to degradation of the instrumental contingency, with electrolytic lesions producing a deficit and NMDA lesions having no apparent effect. Given this difference, it is important to more fully characterize the impact of NMDA lesions on instrumental learning, so the rats were next tested in an outcome devaluation procedure. Electrolytic lesions have been shown previously to have no effect on this form of learning (Corbit and Balleine, 2000); however, the possibility remains that NMDA lesions may produce different effects.

\section{Outcome devaluation}

The results of the devaluation test are illustrated in Figure 6. As suggested by this figure, both the electrolytically and NMDAlesioned groups were sensitive to outcome devaluation and to a similar degree, with both groups performing fewer responses on the lever for which the previously earned outcome had been devalued. Statistical analysis confirms this suggestion, revealing a significant effect of devaluation $\left(F_{(1,17)}=4.763 ; p<0.05\right)$. Although the figure suggests a numerically smaller effect in the NMDA rats, there was no effect of group $(F<1)$ and no devaluation $\times$ group interaction $(F<1)$. These results replicate the previous finding that electrolytic hippocampal lesions have no effect on sensitivity to outcome devaluation and extend this result to NMDA lesions of the hippocampus.

\section{Experiment 4}

\section{Training}

Analysis of the training data revealed that, although the two groups acquired the lever press response in the same number of days, the retrohippocampal rats responded at a higher rate $(\mathrm{M}=$ 31.17; $\mathrm{SE}=2.07)$ at the end of training than the sham rats did
Figure 5. Mean lever presses per minute for electrolytically and NMDA-lesioned rats after degradation of one action-outcome contingency across days of training (left). Right, Lever-press responses for the two groups in a two-lever choice extinction test. Error bars represent \pm 1 SED for the withinsubjects variable in each group.

\section{Group}

\section{Devaluation Extinction Test}

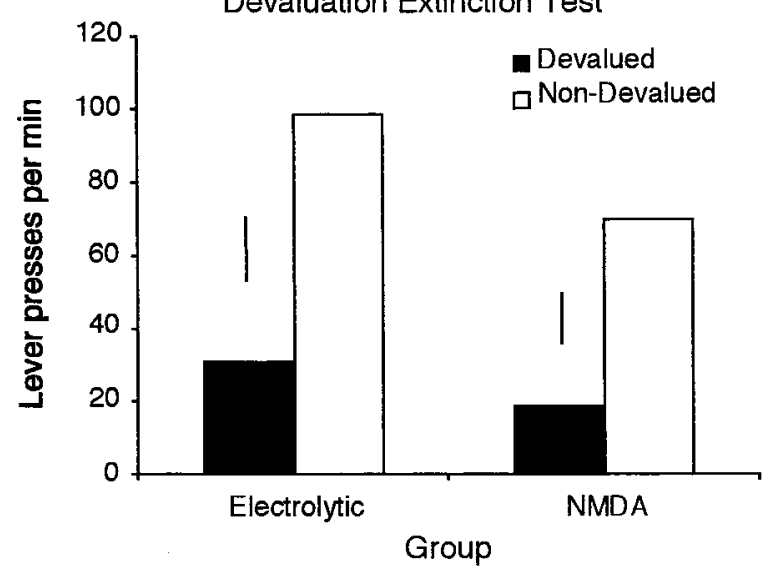

Figure 6. Mean lever presses per minute for electrolytically and NMDAlesioned rats in a two-lever choice extinction test after devaluation of one instrumental outcome. Error bars represent \pm 1 SED for the withinsubjects variable in each group.

$\left(\mathrm{M}=21.57 ; \mathrm{SE}=2.2 ; F_{(1,19)}=9.805 ; p<0.01\right)$. Two sham rats failed to acquire the instrumental response and were excluded from the experiment $(n=9)$.

\section{Contingency degradation training}

Two rats, one from the sham and one from the lesion group, were excluded from analysis of the contingency degradation because of equipment failure. The results from the remaining subjects (sham, $n=8$; retrohippocampal, $n=11$ ) are illustrated in Figure 7, left. Inspection of Figure 7 suggests that, in general, the performance of the sham rats was sensitive to the contingency manipulation. In contrast, the retrohippocampal group responded at generally higher rates and displayed no sensitivity to the contingency manipulation, maintaining similar response rates on the two levers. Confirming this description, the statistical analysis of the contingency training data revealed a significant main effect of contingency in the sham group $\left(F_{(1,17)}=3.33 ; p<0.05\right)$ but no effect of contingency in the retrohippocampal group $(F<1)$, which, if anything, appeared to respond more on the lever with the degraded contingency than on the other lever.

\section{Extinction test}

The test data displayed in Figure 7, right, suggest that the two groups performed very differently in the extinction test. Sham rats 
Figure 7. Mean lever presses per minute across $3 \mathrm{~d}$ blocks for the sham and retrohippocampal lesioned rats after degradation of one action-outcome contingency across days of training (left). Right, Data from a two-lever choice extinction test. Error bars represent \pm 1 SED for the within-subjects variable in each group.
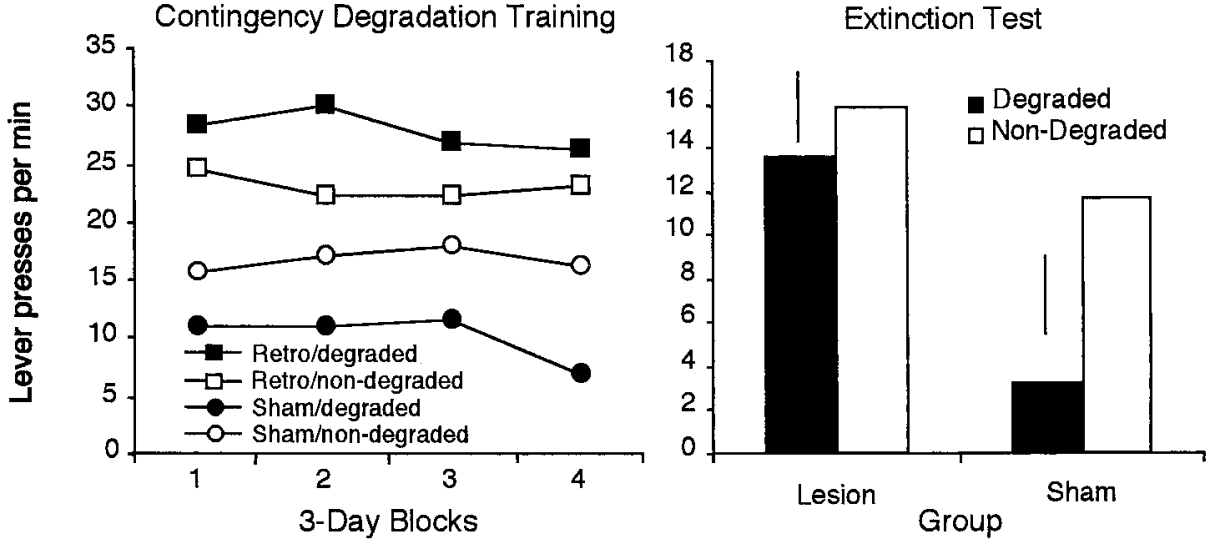

seemed to be sensitive to the contingency manipulation. The retrohippocampal rats, however, responded similarly on the two levers. The statistical analyses were conducted using orthogonal planned comparisons derived from the pattern of results observed during the contingency training phase. These tests established, first, that no contingency effect emerged in the retrohippocampal group; degradation of the instrumental contingency failed to influence performance, and responding was similar on the two levers $\left(t_{(17)}=0.45 ; p>0.05\right)$. In contrast, the sham group was clearly sensitive to degradation of the contingency; thus, although performance did not differ from the retrohippocampal group on the nondegraded action $\left(t_{(17)}=0.70 ; p>0.05\right)$, the responding of the sham rats was reduced selectively and reliably on the lever the response-outcome contingency of which was degraded relative to both the retrohippocampal group and to responding on the nondegraded lever $\left(t_{(17)}=2.58 ; p<0.05\right)$. This pattern of results provides clear evidence that the sham but not the retrohippocampus-lesioned rats were sensitive to degradation of the instrumental contingency, an effect that emerged both during training and in the extinction test.

\section{Devaluation extinction test}

Although the retrohippocampal rats were not sensitive to the contingency degradation, both they and the sham rats displayed sensitivity to outcome devaluation by responding less on the lever that had previously earned the now devalued outcome (lesion, $\mathrm{M}=36, \mathrm{SE}=17$; sham, $\mathrm{M}=12, \mathrm{SE}=4$ ) relative to responding on the other lever (lesion, $\mathrm{M}=182, \mathrm{SE}=37$; sham, $\mathrm{M}=49, \mathrm{SE}=$ 16). Statistical analysis revealed a significant effect of group $\left(F_{(1,19)}=9.48 ; p<0.01\right)$, with retrohippocampal rats performing more responses overall. In addition, there was a significant effect of devaluation $\left(F_{(1,19)}=14.75 ; p<0.01\right)$. Although there appears to be a numerically smaller effect in the sham group, there was no devaluation $\times$ group interaction $\left(F_{(1,19)}=2.59 ; p>0.05\right)$. This result is important, because to perform accurately on this test, rats must be able to discriminate between the two actions and between the two outcomes. The selective performance of the retrohippocampal rats on this test means that neither a failure to discriminate between the two actions nor between the two outcomes can explain their lack of sensitivity to selective degradation of the instrumental contingency described above.

\section{Experiment 5}

\section{Training}

No reliable difference in the rate of acquisition of the lever press response was observed between groups. In addition, by the final day of instrumental training, the unilateral sham $(\mathrm{M}=26.9 ; \mathrm{SE}=$ 3.17), subicular $(\mathrm{M}=31.17 ; \mathrm{SE}=3.70)$, and entorhinal $(\mathrm{M}=$ 27.87; $\mathrm{SE}=4.5)$ groups showed no difference in rate of responding $(F<1)$.

\section{Contingency degradation training}

Figure 8, left, shows the effects of contingency degradation across days of training. As the figure suggests, both unilateral and subiculum-lesioned rats showed sensitivity to the degradation of the instrumental contingency. In contrast, rats with entorhinal lesions responded similarly on both levers, indicating that they were insensitive to the contingency manipulation. Statistical analysis of the degradation training data revealed no effect of group $\left(F_{(2,27)}=1.11 ; p>0.05\right)$, a significant main effect of contingency $\left(F_{(1,27)}=7.32 ; p<0.05\right)$, and a group $\times$ contingency interaction $\left(F_{(2,27)}=4.18 ; p<0.05\right)$. Additional analysis of this interaction revealed a significant simple effect of contingency in the unilateral sham group $\left(F_{(1,9)}=8.51 ; p<0.05\right)$ and the subicular group $\left(F_{(1,9)}=5.14 ; p<0.05\right)$ but not in the entorhinal group $\left(F_{(1,9)}=\right.$ $1.05 ; p>0.05)$.

\section{Extinction test}

Inspection of Figure 8, right, shows that, when tested in extinction, both the unilateral sham and subicular groups responded less on the lever for which the contingency had been degraded, whereas the entorhinal group responded similarly on the two levers. This pattern suggests that the sham and subicular groups but not the entorhinal group were sensitive to the contingency manipulation. Analyses of the extinction data revealed a significant effect of contingency in the sham $\left(F_{(1,9)}=10.94 ; p<0.01\right)$ and subicular $\left(F_{(1,9)}=5.26 ; p<0.05\right)$ groups but not in the entorhinal group $(F<1)$. These results support the claim that the entorhinal cortex but not the subiculum is essential for the detection of instrumental contingency and relate the deficit found after electrolytic lesions of the dorsal hippocampus (experiments 1 and 3) directly to that obtained with neurotoxic lesions of the retrohippocampal complex (experiment 4).

\section{DISCUSSION}

The aim of this study was to characterize further the role of the dorsal hippocampus in instrumental conditioning. Specifically, we sought to replicate the previous finding that electrolytic lesions of the dorsal hippocampus render rats insensitive to the selective degradation of the instrumental action-outcome contingency and to extend this finding by investigating the effects of NMDA lesions of the dorsal hippocampus and of the retrohippocampal 

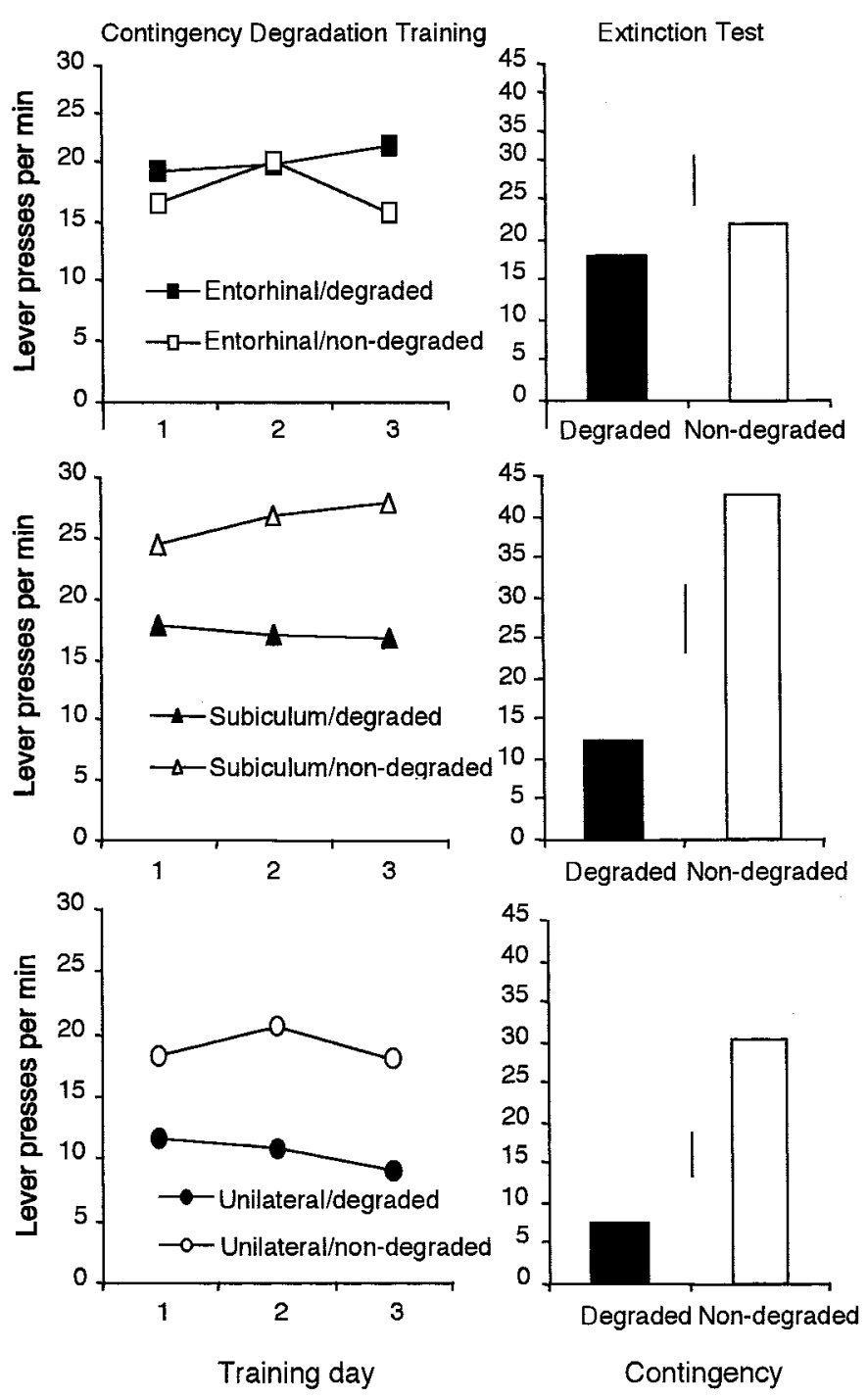

Figure 8. Mean lever presses per minute for the entorhinal (top), subiculum (center), and unilateral sham (bottom) lesioned rats after degradation of one action-outcome contingency across days of training (left). Right, Data from a two-lever choice extinction test. Error bars represent \pm 1 SED for the within-subjects variable in each group.

region (i.e., entorhinal cortex and subiculum). Finally, a disconnection procedure was used to determine more precisely the relative involvement of the entorhinal cortex and subiculum in the detection of changes in the instrumental contingency and to assess whether the deficits observed after cell body lesions of these structures could account for the deficits observed after electrolytic lesions of the dorsal hippocampus.

The results of experiments 1 and 3 confirmed our original observations that electrolytic hippocampal lesions have no effect on the acquisition of instrumental performance but reduce the sensitivity of rats to the selective degradation of the instrumental contingency. Although these results tend to support the suggestion of Devenport and colleagues (Devenport, 1979; Devenport and Holloway, 1980) that the hippocampus is critical for the detection of the causal relationship between an action and outcome, this conclusion is challenged by the results of experiments 2 and 3, which demonstrated that animals with NMDA lesions of the hippocampus were as sensitive as sham-lesioned controls to contingency degradation. As a consequence, these results suggest that the original deficit observed after electrolytic lesions is more likely the result of damage to fibers passing through the region of the lesion.

In our previous analysis (Corbit and Balleine, 2000), we suggested that the deficit in contingency sensitivity induced by electrolytic lesions of the dorsal hippocampus was produced by a reduction in the ability of lesioned animals to calculate the background rate of reinforcement, i.e., the rate of reinforcement in the absence of an action. It is now widely accepted that the reduction in instrumental performance induced by noncontingent outcome delivery is the product of a reduction in the relative validity of the instrumental action compared with the background or context as a predictor of reward (Wagner et al., 1968; Dickinson and Charnock, 1985; Colwill and Rescorla, 1986). As such, and on the basis of reports suggesting that hippocampal lesions induce deficits in context conditioning (Honey and Good, 1993; Maren and Fanselow, 1997), we were led to the suggestion that the failure of hippocampal rats to adjust to degradation of the instrumental contingency was secondary to a deficit in context conditioning. Although we remain convinced that the deficit in contingency degradation observed after electrolytic lesions reflects a deficit in context conditioning, particularly given our repeated failure to find any lesion-induced deficit in outcome devaluation (Corbit and Balleine, 2000), the failure of excitotoxic lesions to replicate this effect suggests that structures outside the dorsal hippocampus are more critically involved in this task.

It is known that NMDA-induced lesions differ from electrolytic lesions in that, although both kill cells in the region of the lesion, the latter also damages axonal fibers of passage (Jarrard, 1993, 1995). It is possible, therefore, that the effects of electrolytic lesions of the dorsal hippocampus in our studies reflect damage to axons from a distal structure involved more directly in context conditioning in this task. There are, in fact, several recent reports that context conditioning is not meditated solely by the hippocampus. For example, in fear conditioning, lesions or inactivation of the major cortical inputs to the hippocampus, including the perirhinal, postrhinal, and entorhinal cortices, produce deficits in freezing conditioned to contextual cues (Corodimas and LeDoux, 1995; Maren and Fanselow, 1997; Sacchetti et al., 1999; Bucci et al., 2000) (but see Phillips and LeDoux, 1995; Bannerman et al., 2001). In addition, lesions of the subiculum, the major source of subcortical output from the hippocampus, disrupt contextual fear conditioning (Maren, 1999). The involvement of these structures in contextual learning suggests that they may play a role in the detection of changes to the instrumental contingency.

Of those areas implicated in contextual learning, striatal projections from the entorhinal cortex (Totterdell and Meredith, 1997) and subiculum (Groenewegen et al., 1987) travel through the alveus, a pathway adjacent to the dorsal hippocampus, before entering the fornix. It is likely, therefore, that the electrolytic hippocampal lesions in experiments 1 and 3 disrupted this pathway, disconnecting circuits originating in the retrohippocampal region.

In experiment 4, combined entorhinal and subicular lesions (i.e., retrohippocampal lesions) were found to render animals insensitive to a change in the instrumental contingency. Furthermore, retrohippocampal rats showed normal sensitivity to selective outcome devaluation, a finding that indicates that the deficit in contingency degradation was not secondary to any failure to discriminate between either the outcomes or the two actions. Importantly, this is exactly the pattern of deficits that was ob- 
served after electrolytic lesions of the dorsal hippocampus (Corbit and Balleine, 2000).

The results of experiment 4 suggest, therefore, that the retrohippocampus is involved in the ability of rats to encode the contingency between actions and their consequences. Nevertheless, these results do not allow us to determine the relative involvement of the entorhinal cortex and subiculum in this effect. In addition, the results of experiment 4 , although suggestive, do not establish definitively that electrolytic lesions of the hippocampus cause their effects by disrupting a pathway arising in the retrohippocampus. A disconnection procedure was used in experiment 5 to identify more clearly the structures involved in the detection of instrumental contingency and to relate the effect of NMDA lesions of the entorhinal cortex and subiculum to the deficits observed after electrolytic lesions of the dorsal hippocampus.

Subjects in experiment 5 all received unilateral electrolytic lesions of the dorsal hippocampus and either sham surgery or NMDA lesions of either the contralateral entorhinal cortex or the subiculum. Although these groups were indistinguishable during the instrumental training phase, only the unilateral sham and subicular groups showed normal sensitivity to the selective degradation of the instrumental contingency. In contrast, the entorhinal group was insensitive to contingency degradation, performing similarly on both levers throughout contingency training and in the extinction test. These results suggest not only that the entorhinal cortex is involved selectively in detecting changes in the contingency between actions and outcomes, but also that the disruption of fibers originating in this structure can account for the effects observed after electrolytic lesions of the dorsal hippocampus.

Although it remains a possibility, as some authors have suggested, that the hippocampus plays a role in contextual discrimination both in the free-operant situation (Freeman et al., 1996) and in response selection in certain tasks (Wise and Murray, 1999), as it stands, these data argue against a direct role for the dorsal hippocampus either in the formation of response-reward associations or in the encoding of the causal relationship between an action and its specific consequences in our instrumental conditioning tasks. Rather, the current experiments suggest that the deficits in the detection of changes in the instrumental contingency that we reported previously after electrolytic lesions were most likely the result of damage to efferents of the entorhinal cortex. It should be noted, however, that the effects of lesions of the ventral hippocampus have not been examined and that the role of this structure remains unknown.

In summary, although electrolytic hippocampal lesions rendered animals relatively insensitive to changes in the instrumental contingency, this deficit did not appear to depend on the integrity of cell bodies within the dorsal hippocampus, because neurotoxic hippocampal lesions produced no detectable deficit. The possibility that the original deficit was secondary to damage to fibers of passage rather than damage to the hippocampus itself was explored by examining the effects of lesions of the retrohippocampal region. These lesions were found to produce a deficit similar to that observed after electrolytic hippocampal lesions with rats failing to adjust their responding after degradation of the actionoutcome contingency. Finally, the deficit observed after NMDA lesions of the entorhinal cortex and electrolytic lesions of con- tralateral dorsal hippocampus supports the claim that it is a circuit originating in the entorhinal cortex and passing through the alveus that is critical for the detection of changes to the instrumental contingency.

\section{REFERENCES}

Balleine BW, Dickinson A (1998) The role of incentive learning in instrumental outcome revaluation by sensory-specific satiety. Anim Learn Behav 26:46-59.

Bannerman DM, Yee BK, Lemaire M, Jarrard L, Iversen SD, Rawlins JN, Good MA (2001) Contextual fear conditioning is disrupted by lesions of the subcortical, but not entorhinal, connections to the hippocampus. Exp Brain Res 141:304-311.

Bucci DJ, Phillips RG, Burwell RD (2000) Contributions of postrhinal and perirhinal cortex to contextual information processing. Behav Neurosci 114:882-894.

Colwill RC, Rescorla RA (1986) Associative structures in instrumental learning. In: The psychology of learning and motivation, Vol 20 (Bower GH, ed), pp 55-104. New York: Academic.

Corbit LH, Balleine BW (2000) The role of the hippocampus in instrumental conditioning. J Neurosci 20:4233-4239.

Corodimas KP, LeDoux JE (1995) Disruptive effects of post-training perirhinal cortex lesions on conditioned fear: contributions of contextual cues. Behav Neurosci 109:613-619.

Devenport LD (1979) Superstitious bar pressing in hippocampal and septal rats. Science 205:721-723.

Devenport LD (1980) Response-reinforcer relations and the hippocampus. Behav Neural Biol 29:105-110.

Devenport LD, Holloway FA (1980) The rat's resistance to superstition: role of the hippocampus. J Comp Physiol Psychol 4:691-705.

Dickinson A, Balleine BW (1994) Motivational control of goal-directed action. Anim Learn Behav 22:1-18.

Dickinson A, Charnock DJ (1985) Contingency effects with maintained instrumental reinforcement. Q J Exp Psychol 37B:397-416.

Freeman JH, Cuppernell C, Flannery K, Gabriel M (1996) Contextspecific multi-site cingulate cortical, limbic thalamic and hippocampal neuronal activity during concurrent discriminative approach and avoidance training in rabbits. J Neurosci 16:1538-1549.

Groenewegen HJ, Vermeulen-Van der Zee E, te Kortschot A, Witter MP (1987) Organization of the projections from the subiculum to the ventral striatum in the rat: a study using anterograde transport of Phaseolus vulgaris leucoagglutinin. Neuroscience 23:103-120.

Hammond LJ (1980) The effect of contingency upon the appetitive conditioning of free-operant behavior. J Exp Anal Behav 34:297-304.

Honey RC, Good M (1993) Selective hippocampal lesions abolish the contextual specificity of latent inhibition and conditioning. Behav Neurosci 107:23-33.

Jarrard LE (1993) On the role of the hippocampus in learning and memory in the rat. Behav Neural Biol 60:9-26.

Jarrard LE (1995) What does the hippocampus really do? Behav Brain Res 71:1-10.

Maren S (1999) Neurotoxic or electrolytic lesions of the ventral subiculum produce deficits in the acquisition and expression of Pavlovian fear conditioning in rats. Behav Neurosci 113:283-290.

Maren S, Fanselow MS (1997) Electrolytic lesions of the fimbria/fornix, dorsal hippocampus, or entorhinal cortex produce anterograde deficits in contextual fear conditioning in rats. Neurobiol Learn Mem 67:142-149.

Paxinos G, Watson C (1998) The rat brain in stereotaxic coordinates. San Diego: Academic.

Phillips RG, LeDoux JE (1995) Lesions of the fornix but not the entorhinal or perirhinal cortex interfere with contextual fear conditioning. J Neurosci 15:5308-5315.

Sacchetti B, Lorenzini CA, Baldi E, Tassoni G, Bucherelli C (1999) Auditory thalamus, dorsal hippocampus, basolateral amygdala, and perirhinal cortex role in the consolidation of conditioned freezing to context and to acoustic conditioned stimulus in rats. J Neurosci 19:9570-9578.

Totterdell S, Meredith GE (1997) Topographical organization of projections from the entorhinal cortex to the striatum of the rat. Neuroscience 78:715-729.

Wagner AR, Logan FA, Haberlandt K (1968) Stimulus selection in animal discrimination learning. J Exp Psychol 76:177-186.

Wise SP, Murray EA (1999) Role of the hippocampal system in conditional motor learning: mapping antecedents to action. Hippocampus 9:101-117. 